\title{
Movement and Imaging of Single-Atom Dopants in Silicon
}

\author{
Bethany M. Hudak ${ }^{1}$, Jiaming Song ${ }^{1}$, Paul C. Snijders ${ }^{1}$, and Andrew R. Lupini ${ }^{1}$ \\ 1. Materials Science and Technology Division, Oak Ridge National Laboratory, Oak Ridge, TN, USA
}

Theoretical predictions show that quantum computers should be able to perform beyond the capabilities of the most powerful current supercomputers, making the realization of quantum computing of interest to both civilian and government institutions. One quantum computing architecture that is particularly appealing consists of individual atoms doped into a semiconductor, where the spin states of these dopant atoms provide a method to encode the qubits [1]. The widespread application of silicon in electronics makes it an ideal material for quantum computing due to existing infrastructure for the production, study, and use of Si-based devices. Group V elements are promising candidates for use as single-atom qubit dopants in $\mathrm{Si}[1,2]$. Atoms with a similar atomic number $(\mathrm{Z})$ to $\mathrm{Si}$, such as phosphorous, are difficult to observe through conventional single-atom imaging techniques such as scanning transmission electron microscopy (STEM), making approaches to accurate single atom positioning challenging. Pnictogens other than phosphorous, particularly the heavier Group V elements, are potential candidates to function as qubits and overcome these obstacles. Bismuth - with large spin-orbit coupling, strong clock transitions [3], and a greater potential to be imaged by STEM - is very promising. Bi in Si has been shown theoretically to allow gates operating in the $\mathrm{MHz}$ regime [4]. It has also been suggested that Bi may allow qubit operation at liquid nitrogen temperature, an important aspect of practical device operation.

However, the accurate placement of single atom dopants within a solid with control and understanding of their local environment has not yet been realized with the necessary degree of accuracy for quantum computing applications. Here we demonstrate the ability to image and move single $\mathrm{Bi}$ atoms within Si. Samples of Bi-doped Si films were grown using molecular beam epitaxy (MBE) and imaged at a variety of accelerating voltages. The dopant atoms can be imaged with atomic-resolution at low voltages, and at higher voltages we intentionally induce the motion of single $\mathrm{Bi}$ dopants inside the film. Figure 1 shows a selection of frames from a sequence of images taken at $200 \mathrm{kV}$ demonstrating that under these conditions we can observe the movement of a single $\mathrm{Bi}$ dopant. Image sequences acquired at lower accelerating voltages show less dopant movement.

Electron beam interactions with the sample are well known. Recently, it has been shown that the electron beam can be used for atomic-level sculpting [5] of oxide lattices. While this phenomenon may be somewhat expected, the mechanism by which it occurs needs to be understood so that the process can be controlled and exploited. Due to the dopant behavior above and below the knock-on damage threshold of $\mathrm{Si}$, and with the use of density functional theory (DFT) calculations, we have determined some of the factors that are necessary to produce dopant movement. By fully understanding the mechanism by which the dopant atoms move and developing this mechanism for directed atom movement, we can begin to build atomic-scale architectures for quantum computing. [6] 


\section{References:}

[1] BE Kane, Nature 393 (1998), p. 133-137.

[2] JJL Morton et al, Nature 455 (2008), p. 1085-1088.

[3] G Wolfowicz et al, Nat. Nanotechnol. 8 (2013), p. 561-564.

[4] G Pica et al, Phys. Rev. B. 90 (2014), p. 195204.

[5] S Jesse et al, Small 11 (2015), p. 5895-5900.

[6] Research sponsored by the Laboratory Directed Research and Development Program of Oak Ridge National Laboratory, managed by UT-Battelle, LLC, for the U. S. Department of Energy.

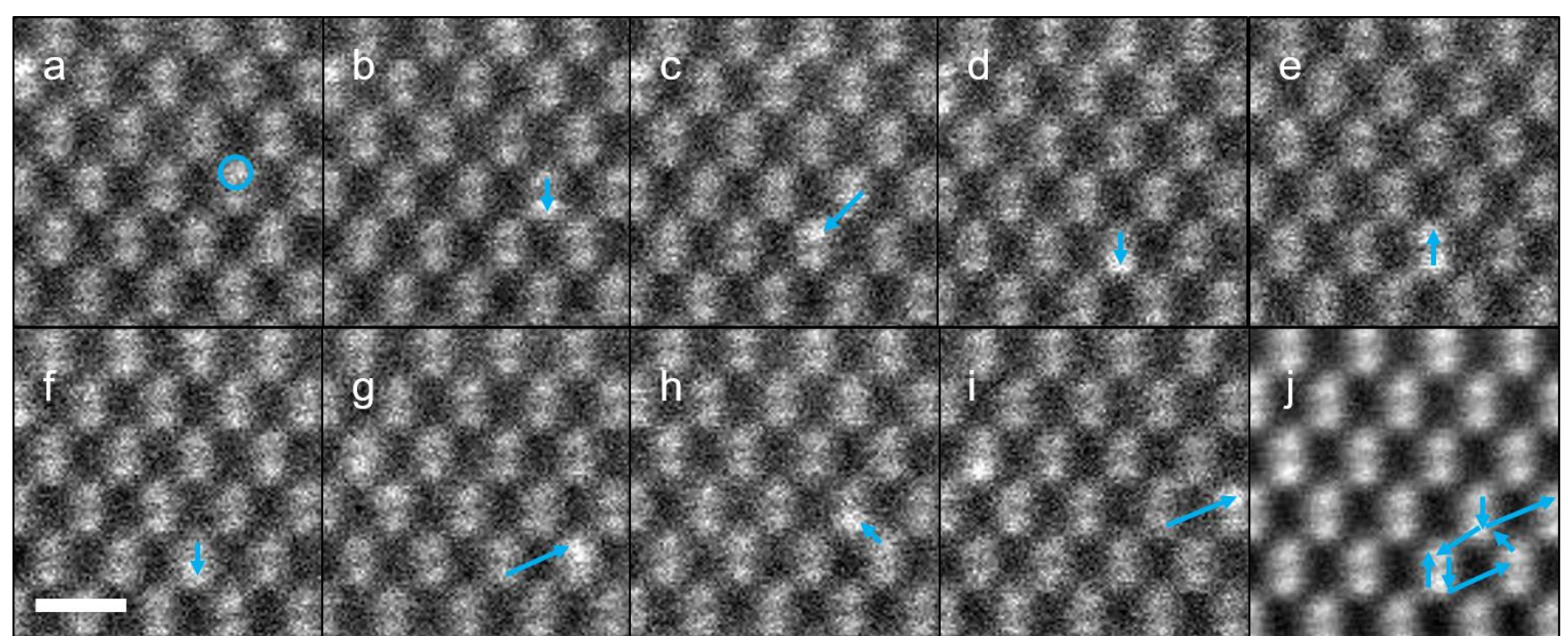

Figure 1. Electron beam-induced movement of a single Bi atom. (a-i) Extract from 20 sequential frames acquired at $200 \mathrm{kV}$. (a-i) The random motion of one $\mathrm{Bi}$ atom is followed, as indicated by arrows. (j) Overlaid images showing the path covered by the $\mathrm{Bi}$ atom during this acquisition. Beam current was about $140 \mathrm{pA}$ with an $8 \mathrm{~nm}$ field of view at $4 \mu \mathrm{S} /$ pixel. Scale bar $=0.5 \mathrm{~nm}$ 